\title{
Effects of essence oil additives added to different feeds on methane production
}

\section{Efectos sobre la producción de metano de los aceites esencias añadiendo diferentes aditivos}

\author{
Ahmet Tekeli ${ }^{*}$ Ph.D, Gültekin Yıldız² Ph.D, Winfried Drochner ${ }^{3}$ Ph.D, \\ Herbert Steingass, ${ }^{3}$ Ph.D.
}

\begin{abstract}
${ }^{1}$ Yuzuncu Yıl University, Faculty of Agriculture, Department of Animal Science, 65080, Van//Turkey. 2 Ankara University, Faculty of Veterinary, Department of Animal Nutrition and Nutritional Diseases, 06110 Ankara/Turkey. ${ }^{3}$ Hohenheim University, Department of Animal Nutrition, 70599 Stuttgart/ Germany. *Corresponding: atekeli@yyu.edu.tr
\end{abstract}

Received: January 2016; Accepted: December 2016.

\begin{abstract}
Objective. The aim of the study is to determine the effect of different plant essence oil supplementation to TMR, concentrate and hay on methanogenesis and at 2, 8 and 24 hours (h) using in vitro gas production technique in cattle. Material and methods. Three fistulated Holstein dairy cows were used for rumen fluid collection for application of in vitro gas production technique. Four essence oils ( $T$. vulgaris, $O$. vulgare, S. aromaticum, Z. officinale) were used as plant extracts. Results. Essence oil supplementation, particularly at increased doses, significantly decreased methane production for TMR, concentrate and hay at 8 and $24 \mathrm{~h}$ compared to negative and positive control groups $(\mathrm{p}<0.05)$. Among all plant extracts, Syzygium 200 ppm supplementation resulted the lowest methane production values $(p<0.05)$. While methane production at $24 \mathrm{~h}$ in positive control groups were recorded respectively as $10.45,10.75$ and 10.07 for TMR, concentrate and hay, the values in Syzygium 200 ppm group were recorded respectively as $1.65,3.28$ and 1.98 for these feed groups. Conclusions. The findings of the study indicate that increased doses of essence oil supplementation significantly decrease methane production in ruminants. Hence, it is suggested that essence oil will be highly beneficial in ruminant nutrition.
\end{abstract}

Keywords: Aromatic plants, essence oil, methane production, ruminant nutrition (Source: CAB).

\section{RESUMEN}

Objectivo. El objectivo del presente estudio es determin que el efecto de diferentes suplementos de aceite de esencia de la planta es de TMR, concentrado y heno en la metanogénesis ya los 2,8 y 24 horas (h) utilizando la técnica in vitro de producción de gas en los bovinos. Material y métodos. Tres vacas lecheras Holstein fistulados se utilizaron para la acumulación de liquido ruminal para la aplicación de la técnica in vitro de producción de gas en. Cuatro aceites esenciales (T.vulgaris, O.vulgare, S.aromaticum, Z.officinale) fueron utilizadas como extractos de plantas. Resultados. La administración de suplementos de aceite de esencia, particularmente a elevadas dosis, disminoyó significativamente la producción de metano de TMR, se concentran y heno a las 8 y las $24 \mathrm{~h}$ en comparación con los grupos negativos y positivos de control $(p<0.05)$. Entre todos los extractos de plantas, Syzgium 200 suplementación ppm de corrientes de aire los valores de producción de metano màs bajas $(p<0.05)$. Mientras que la producción de metano a las $24 \mathrm{~h}$ en los grupos de control positivos, respectivamente, como $10.45,10.75$ y 10.07 am para TMR, concentrarse y heno, los valores de Syzygium grupo de 200 
ppm se registraron, respectivamente $1.65,3.28$ y 1.98 para los thes grupos alimentar. Conclusiones: Los resultados del estudio indican que las dosis elevadas de suplementos de aceite de esencia para disminuir significativamanente la producción de metano en rumiantes. Por lo tanto, se sugiere que el aceite de esencia será altamente beneficioso en la nutrición de rumiantes.

Palabras clave: Aceite esencial, plantas aromáticas, nutrición de rumiantes (Fuente: CAB).

\section{INTRODUCTION}

Ionophore antibiotics have been successfully used in rumens for many years to reduce energy (methane emission) and protein (ammonia nitrogen emission) losses (1). While ionophore antibiotics have been widely used in conventional production systems for the control of rumen fermentation, the European Commission (EC) decided to phase out, and ultimately ban (January 1st 2006), the use of antibiotics in feed (70/524/EEC Directive and EC Regulation No:1831/2003)(2). Hence, alternative substances were started to be explored for antibiotics. One of these possible alternatives are the plant extracts, which are also called as essence oils. The term essential oil is misleading as they are not -as their name suggests- 'essential' for nutrition or metabolism nor are they oils in the sense of being glycerol based lipids. Thus, it would be more appropriate to use the terms "plant extract" or "essence oil" instead of the term "essential oil".

Hart et al (3) have been reported that plant extracts decreased methane production with a subsequent decrease in rumen methanogens. Methane is one of the most important greenhouse gases that contribute to global warming. The population of ruminants is an important source of methane, contributing approximately $15 \%$ of the total atmosferic methane flux (4). Despite the greenhouse effect of methane at a life-threatening rate, no chemicals with long-term effects that would prevent methane production could have been developed so far. The only method used for reducing methane production is suppressing the number of microorganisms consuming $\mathrm{H}_{2}$ - which is used in methane synthesis- by using antibiotics or ionophores. Nevertheless, this method has resulted only a $25 \%$ reduction in methane production (5). Takashi (6) reported that methane production from ruminants in the developing countries may be high since their diets are often lack of critical nutrients for efficient microbial growth in the rumen. Plant extracts have been reported to decrease methane production with a subsequent

\section{INTRODUCCIÓN}

Los antibióticos ionóforos se han utilizado en rúmenes exitosamente durante varios años para reducir la pérdida de energía (emisión de metano) y de proteína (emisión de nitrógeno amoniacal) (1). Mientras que los antibióticos ionóforos han sido ampliamente utilizados en sistemas de producción convencionales para controlar la fermentación ruminal, la Comisión Europea decidió eliminar y, en última instancia, prohibir el uso de antibióticos en piensos (70/524/ CEE Directiva y Reglamento de la CE no 1831/2003)(2). Por ello dio inicio la explorarión sustancias alternativas para ser usadas en antibióticos. Una de estas posibles alternativas son los extractos vegetales, también denominados aceites esenciales. El término aceite esencial es engañoso, ya que no son -como su nombre indicaesenciales para la nutrición o el metabolismo, ni son aceites en el sentido de ser lípidos a base de glicerol. Por lo tanto sería más apropiado utilizar los términos «extracto vegetal» 0 «aceite de esencias» " en lugar del término "aceite esencial".

Hart et al (3) reportaron que los extractos vegetales disminuyeron la producción de metano, con una posterior disminución en los metanógenos del rumen. El metano es uno de los gases de efecto invernadero más importantes que contribuye al calentamiento global. La población de rumiantes es una fuente importante de metano, que aporta aproximadamente el $15 \%$ de la totalidad del flujo atmosférico de metano (4). A pesar del efecto invernadero causado por el metano, a una tasa potencialmente mortal, ningún producto químico con efectos que pudieran impedir la producción de metano a largo plazo pudo ser desarrollado hasta el momento. El único método utilizado para reducir la producción de metano es suprimir el número de microorganismos que consumen $\mathrm{H}_{2}$ - utilizado en la síntesis del metano - mediante el uso de antibióticos o ionóforos. Sin embargo, este método sólo ha logrado una reducción del $25 \%$ en la producción de metano (5). Takashi (6) reportó que la producción de metano en rumiantes de países en vía de desarrollo puede ser alta, debido a que en sus dietas tiende a haber una falta de nutrientes que resultan críticos para un crecimiento microbiano eficiente en el rumen. Se ha reportado que los extractos vegetales disminuyen la producción de metano 
decrease in rumen methanogens (3). One of the most important goals of stock breeders is manipulation of rumen microbial ecosystem to improve ruminant performance and to reduce methane emissions generated by ruminants. Methane is one of the most important greenhouse gases that contribute to global warming. Besides constituting a significant loss of energy for the animal, methane is a very potent greenhouse gas since it is given off atmosphere (7). Methane is 23 times more potent as a greenhouse gas than carbon dioxide (8). The global population of ruminants in 2014 was reported as 3.875 billion (9). Global ruminant population is significantly growing accompanied with significant increase in atmospheric methane concentrations. There is a strong correlation between increasing atmospheric methane concentrations and growing global ruminant population (10).

Feeding strategies are required to reduce methane production generated by ruminants. One of the alternative feed supplements are plant extracts. In this study, Oreganum vulgare, Thymus vulgaris, Zingiber officinale, Syszygium aromaticum essence oils were supplemented to TMR, concentrate and hay to explorepotential capacities of these essence oils in reducing methane productionat 2,8 and $24 \mathrm{~h}$ in in vitro conditions. The aim of the study is to determine the effect of different plant essence oil supplementation to TMR, concentrate and hay on methanogenesis and at 2, 8 and 24 hours (h) using in vitro gas production technique in cattle.

\section{MATERIALS AND METHODS}

Animals and feeds. Three fistulated Holstein dairy cows were used for rumen fluid collection for application of in vitro gas production technique. Fistulated diary cows were fed according to the caring level with a diet containing alfalfa hay $(\% 60)$ and concentrate (\%40) twice a day. For each feed (TMR, Concentrate and Hay), ruminal fluid samples were collected twice day on different days. Four essence oils ( $T$. vulgaris, $O$. vulgare, $S$. aromaticum, $Z$. officinale) were used as plant extracts. T. vulgaris, S. aromaticum, and $Z$. officinale essence oils were obtained from Ege Lokman San. Tic. Company in Manisa Province (Turkey) and $O$. vulgare essence oil from Aksu Gida San. Tic. Company in Mersin Province (Turkey). All plant extracts were extracted with distilated water. For each extract, different con una posterior disminución en los metanógenos del rumen (3) Uno de los objetivos importantes de los ganaderos es la manipulación del ecosistema microbiano ruminal para mejorar el rendimiento en rumiantes y reducir las emisiones de metano generadas por los rumiantes. El metano es uno de los más importantes gases de efecto invernadero que contribuye al calentamiento global. Además de constituir una pérdida de energía significativa para el animal, el metano es un gas de efecto invernadero muy potente puesto que se da por fuera de la atmósfera (7). El metano es 23 veces más poderoso para el efecto invernadero que el dióxido de carbono (8). En 2014 se reportó que la población mundial de rumiantes ascendió a los 3.875 millones (9). La población mundial de rumiantes está creciendo significativamente junto con un aumento significativo en las concentraciones atmosféricas de metano. Existe una fuerte correlación entre el aumento en las concentraciones atmosféricas de metano y la creciente población mundial de rumiantes (10).

Se requieren estrategias de alimentación orientadas a reducir la producción de metano generada por rumiantes. Los extractos vegetales están entre los suplementos alimenticios alternativos. En este estudio, los aceites esenciales de Oreganum vulgare, Thymus vulgaris, Zingiber officinale $y$ Syszygium aromaticum fueron utilizados como suplemento en la ración total mezclada (TMR), el concentrado y el heno para explorar las potenciales capacidades de reducción en la producción de metano de dichos aceites esenciales a 2, 8 y $24 \mathrm{~h}$ en condiciones in vitro. El objetivo de este estudio fue determinar el efecto sobre la metanogénesis de los distintos suplementos a base de aceites esenciales en la ración total mezclada (TMR), el concentrado y el heno, a las 2,8 y 24 horas (h) utilizando una técnica de producción de gas in vitro en bovinos.

\section{MATERIALES Y MÉTODOS}

Animales y piensos. Se usaron tres vacas lecheras Holstein fistuladas para la recolección de líquido ruminal para ser aplicado en la técnica de producción de gas in vitro. Las vacas lecheras fistuladas fueron alimentadas conforme al nivel de cuidado por medio de una dieta con contenido de alfalfa (\%60) y concentrado (\%40), suministrada dos veces al día. Para cada pienso (TMR, concentrado y heno), se recogieron muestras de líquido ruminal dos veces por día en distintos días. Se utilizaron cuatro aceites esenciales como extractos vegetales ( $T$. vulgaris, $O$. vulgare, $S$. aromaticum, $Z$. officinale). Los aceites esenciales $T$. vulgaris, $S$. aromaticum y $Z$. officinale se obtuvieron de la empresa Ege Lokman San. Tic., ubicada en la provincia de Manisa (Turquía) y el aceite de esencias $O$. vulgare se 
doses were tested to determine harmful and usable doses. The samples were incubated in vitro rumen fluid in calibrated glass syringes. The $200 \mathrm{mg}$ samples were weighed into calibrated glass syringes of $100 \mathrm{ml}$. The syringes were pre-warmed at $39^{\circ} \mathrm{C}$ before the injection of $30 \mathrm{ml}$ rumen fluid-buffer mixture into each syringe followed by incubarion in a water bath at $39^{\circ} \mathrm{C}$. Incubation was run for each regulation in 2, 8 and $24 \mathrm{~h}$ time periods. Total mixed ration (TMR), concentrate and hay were used as substrates. In the 2, 8 and $24 \mathrm{~h}$ of the experiment methane levels were measured.

The group with only rumen liquor was assigned as the negative control group and the group with rumen fluid and feed samples was assigned as the positive control group. The groups with rumen fluid, feed samples and thymol, oregano, zingiber and syzygium essence oils were the treatment groups. The compositions of TMR, concentrate and hay used in the experiment are presented respectively in table 1 . The effects of Syzygim aromaticum, Zingiber officinale, Thymus vulgaris, Oreganum vulagare supplementation to TMR, concentrate and hay on methanogenesis production were assessed in the present experiment by incubation of in buffered rumen fluid using an in vitro gas production technique (HFT).

Table 1. Composition of TMR, Concentrate and Alfalfa hay.

\begin{tabular}{cccc}
\hline & Dry matter & Crude protein & MJNEL/kg DM \\
\hline TMR* & 43.00 & 15.25 & 6.9 \\
Concentrate $* *$ & 98.50 & 20.30 & 8.7 \\
Alfalfa Hay & 95.31 & 14.46 & 8.0 \\
\hline
\end{tabular}

*TMR composition: Maize silage $28.9 \%$, Grass silage $31.2 \%$, Hay $4.8 \%$, Haylage $2.3 \%$, Molassed sugar beet pulp $1.3 \%$, Josera-betavit $0.09 \%$, Salt $0.09 \%$, Monosodium phosphate $0.09 \%$, Ca-carbonate $0.35 \%$, Bergophor GM13 0.45\%, Bergophor LM07 0.45\%, MgO 0.02\%, Concentrate-TMR-silage- $2,17.4 \%$, Soybean meals $0.7 \%$, chelates $0.3 \%$, Water $12.1 \%$, Yeast mixture $100 \mathrm{~g}$. Mineral g/kg DM: Ca 7.62, P 4.53, Mg 2.52, Na 2.82, K 16.04, Zn 62, Mn 58 and Se 0.1 .

**Concentrate composition: Maize $20 \%$, sunflower $7.5 \%$, pea $8 \%$, barley $16 \%$, soyabean meal $15 \%$, wheat $30.5 \%$, crib bean $3 \%$.

Methane measurements in in vitro gas production study. Methane measurement depending on experimental and in vitro gas production of study has been carried out at Animal Science Department of Hohenheim University, Germany. Methane concentration was measured in the gas produced during 2, 8 and $24 \mathrm{~h}$ incubation by methane gasmitter ${ }^{\circledR}$ (AGM30/CH4; Firma PRONOVA Analysentechnik $\mathrm{GmbH} \& \mathrm{Co}$; measurement range 0 - $5 \mathrm{Vol} . \%$ ). obtuvo de la empresa Aksu Gıda San. Tic., ubicada en Mersin (Turquía). Todos los extractos vegetales fueron extraídos con agua destilada. Se realizaron ensayos de cada extracto utilizando distintas dosis con el fin de determinar cuáles dosis son nocivas y cuáles son utilizables. Las muestras se incubaron en fluido ruminal in vitro en jeringas de vidrio calibrado. Las muestras de 200 mg fueron pesadas para ser trasladadas a jeringas calibradas de vidrio de 100 $\mathrm{ml}$. Se precalentaron las jeringas a $39^{\circ} \mathrm{C}$ antes de la inyección con una mezcla de fluido de rumentampón en cada jeringa, seguido de una incubación en baño de agua a $39^{\circ} \mathrm{C}$. La incubación se llevó a cabo en períodos de tiempo de 2, 8 y $24 \mathrm{~h}$ para cada regulación. La ración total mezclada (TMR), el concentrado y el heno fueron utilizados como sustratos. Los niveles de metano fueron medidos a las 2,8 y $24 \mathrm{~h}$ del experimento.

Se asignó grupo de control negativo al grupo que sólo utilizó rumen, y se asignó grupo de control positivo al grupo que utilizó fluido ruminal y muestras de pienso. Los grupos de tratamiento fueron grupos que utilizaron fluido ruminal, muestras de piensos y aceites de timol, orégano, zingiber y esencia de syzygium. Las composiciones de ración total mezclada, concentrado y heno utilizadas en el experimento se muestran respectivamente en la tabla 1. En el presente experimento se evaluaron los efectos de la suplementación con Syzygim aromaticum, Zingiber officinale, Thymus vulgaris, y Oreganum vulgare a la masa mexclada total, el concentrado y el heno y su efecto sobre la producción metanogenética por medio de la incubación de fluido ruminal tamponado, para lo cualse utilizó una técnica de producción de gas in vitro (HFT).

\section{Mediciones de metano en el estudio de producción de gas in vitro. La medición de metano según la producción de gas experimental e in vitro del presente estudio se llevó a cabo en el Departamento de Ciencias Animales de la Universidad de Hohenheim, Alemania. Se midió la concentración de metano del gas producido durante la incubación de 2, 8 y 24 horas por medio de un Gasmitter ${ }^{\circledR}$ (AGM30 / CH4, Firma PRONOVA Analysentechnik $\mathrm{GmbH} \& \mathrm{Co}$, con un rango de medición de 0-5 Vol.\%).}

Análisis estadístico. Las estadísticas descriptivas de los parámetros bajo examen se expresaron en términos de errores medios y estándar. Se realizó un Análisis de Varianza Factorial (ANOVA Factorial) para determinar las diferencias entre los medios de suplementos y tipos de pienso según los parámetros examinados. Además, se realizó un ANOVA de Medidas Repetidas para determinar las diferencias relativas a tipos de pienso y duración (horas). Tras los análisis de varianza, se realizó la prueba de Tukey 
Statistical analysis. The descriptive statistics for the examined parameters were expressed in terms of average and standard errors. Factorial Analysis of Variance (Factorial ANOVA) was conducted to determine any differences between the means of supplements and feed types with respect to the examined parameters. Additionally, Repeated Measures ANOVA was performed to determine any differences with respect to feeds and durations (hours). Following analyses of variance, Tukey test was conducted to determine varying means (11). Statistical significance level was set at $5 \%$ and calculations were performed by SPSS Ver13 statistical software package.

\section{RESULTS}

Descriptive statistics and comparative results on methane production (\%) for feed groups according to time period and supplement groups are presented in table 2 . Changes in methane production (\%) at 2, 8 and $24 \mathrm{~h}$ with essence oil supplementation for TMR, concentrate and hay are depicted in figures 1 , 2 and 3 , respectively.

Methane production values significantly decreased in time in all treatment groups for all feeds $(p<0.05)$. Methane production values for TMR negative control and TMR positive control para determinar los distintos medios (11). El nivel de significación estadística se estableció en $5 \%$ y los cálculos fueron realizados utilizando el paquete de software estadístico SPSS Ver.13.

\section{RESULTADOS}

Las estadísticas descriptivas y los resultados comparativos sobre la producción de metano (\%) se muestran en la Tabla 2 por grupos de piensos y según el período de tiempo y el grupo de suplemento. Los cambios en la producción de metano (\%) a las 2, 8 y 24 horas con la suplementación de aceites esenciales en la TMR, el concentrado y heno, están representados en las figuras 1,2 y 3 , respectivamente.

Los valores de producción de metano disminuyeron significativamente en el tiempo a lo largo de todos los grupos de tratamiento y con todos los piensos $(p<0.05)$. Los valores de producción de metano de control negativo y control positivo de TMR a las $24 \mathrm{~h}$ fueron de 12,34 y 10,45, respecticamente, mientras que el menor valor de producción de metano (1.65) se registró en el grupo TMR con suplementación de Syzygium $200(p<0.5)$. Para el grupo de concentrados, los valores de producción de metano a las $24 \mathrm{~h}$ disminuyeron significativamente en la suplementación con Thymol 25, Oregano 25, Zingiber 200 y Syzygium 200 en comparación con los grupos control negativo y control positivo $(p<0.05)$.

Table 2. Descriptive statistics and comparative results on methane production (\%) for feed Groups according to time period and supplement groups

\begin{tabular}{|c|c|c|c|c|c|c|c|c|c|c|c|c|c|c|c|c|}
\hline \multirow{2}{*}{$\begin{array}{l}\text { Group } \\
\text { Feed }\end{array}$} & \multicolumn{2}{|c|}{$\begin{array}{l}\text { Negative } \\
\text { control }\end{array}$} & \multicolumn{2}{|c|}{$\begin{array}{c}\text { Positive } \\
\text { control }\end{array}$} & \multicolumn{2}{|c|}{ Thymol 12.5} & \multicolumn{2}{|c|}{ Thymol 25} & \multicolumn{2}{|c|}{ Oregano 12.5} & \multicolumn{2}{|c|}{ Oregano 25} & \multicolumn{2}{|c|}{ Zingiber 200} & \multicolumn{2}{|c|}{ Syzygium 200} \\
\hline & $\bar{x}$ & $\mathbf{S}_{\bar{x}}$ & $\bar{x}$ & $\mathbf{S}_{\bar{x}}$ & $\bar{x}$ & $\mathbf{S}_{\bar{x}}$ & $\bar{x}$ & $\mathbf{S}_{\bar{x}}$ & $\bar{x}$ & $\mathbf{S}_{\bar{x}}$ & $\bar{x}$ & $\mathbf{S}_{\bar{x}}$ & $\bar{x}$ & $\mathbf{S}_{\bar{x}}$ & $\bar{x}$ & $\mathbf{S}_{\bar{x}}$ \\
\hline TMR2 & $\begin{array}{l}0.00 \\
\text { ec1 }\end{array}$ & 0.00 & $\begin{array}{c}14.76 \\
\text { aA } 1\end{array}$ & 0.46 & $\begin{array}{c}13.85 \\
\text { aA1 }\end{array}$ & 0.38 & $\begin{array}{c}12.57 \\
\text { bA1 }\end{array}$ & 0.59 & $\begin{array}{c}12.48 \\
\mathrm{bA} 1\end{array}$ & 0.91 & $\begin{array}{c}12.30 \\
\text { bA1 }\end{array}$ & 1.11 & $\begin{array}{c}10.68 \\
\text { CA3 }\end{array}$ & 0.46 & $\begin{array}{l}8.42 \\
d A 3\end{array}$ & 0.14 \\
\hline TMR8 & $\begin{array}{c}11.03 \\
\mathrm{aB} 2\end{array}$ & 0.28 & $\begin{array}{l}9.03 \\
b C 1\end{array}$ & 0.15 & $\begin{array}{l}9.50 \\
b C 1\end{array}$ & 0.19 & $\begin{array}{l}8.90 \\
\text { bB1 }\end{array}$ & 0.40 & $\begin{array}{l}9.42 \\
\text { bB1 }\end{array}$ & 0.23 & $\begin{array}{l}9.18 \\
\text { bB1 }\end{array}$ & 0.24 & $\begin{array}{l}8.57 \\
\text { bB1 }\end{array}$ & 0.04 & $\begin{array}{l}6.32 \\
\text { cB1 }\end{array}$ & 0.18 \\
\hline TMR24 & $\begin{array}{c}12.34 \\
\mathrm{aA} 1\end{array}$ & 0.09 & $\begin{array}{c}10.45 \\
\text { bB1 }\end{array}$ & 0.18 & $\begin{array}{c}10.82 \\
\text { bB1 }\end{array}$ & 0.39 & $\begin{array}{l}9.43 \\
\text { bcB1 }\end{array}$ & 0.17 & $\begin{array}{c}10.30 \\
\text { bB1 }\end{array}$ & 0.17 & $\begin{array}{l}9.77 \\
\text { bcB1 }\end{array}$ & 0.37 & $\begin{array}{l}8.52 \\
\mathrm{cB} 12\end{array}$ & 0.23 & $\begin{array}{l}1.65 \\
\mathrm{dC} 1\end{array}$ & 0.23 \\
\hline Con2 & $\begin{array}{l}0.00 \\
\text { CB1 }\end{array}$ & 0.00 & $\begin{array}{c}13.47 \\
\mathrm{aA} 1\end{array}$ & 0.75 & $\begin{array}{c}13.67 \\
\mathrm{aA} 1\end{array}$ & 0.49 & $\begin{array}{c}13.16 \\
\text { aA1 }\end{array}$ & 0.51 & $\begin{array}{c}12.95 \\
\mathrm{aA} 1\end{array}$ & 0.40 & $\begin{array}{c}13.32 \\
\text { aA1 }\end{array}$ & 0.66 & $\begin{array}{c}13.08 \\
\mathrm{aA} 2\end{array}$ & 0.68 & $\begin{array}{c}11.42 \\
\text { bA2 }\end{array}$ & 0.99 \\
\hline Con8 & $\begin{array}{c}10.69 \\
\mathrm{aA} 2\end{array}$ & 0.63 & $\begin{array}{c}9.98 \\
\mathrm{abB} 1\end{array}$ & 0.38 & $\begin{array}{l}10.47 \\
\text { abB1 }\end{array}$ & 0.58 & $\begin{array}{l}8.43 \\
\mathrm{cdB} 1\end{array}$ & 0.13 & $\begin{array}{l}9.20 \\
\text { bcC1 }\end{array}$ & 0.21 & $\begin{array}{l}7.30 \\
\mathrm{~dB} 2\end{array}$ & 0.29 & $\begin{array}{l}8.52 \\
\text { cdB1 }\end{array}$ & 0.18 & $\begin{array}{l}5.78 \\
\text { eB1 }\end{array}$ & 0.23 \\
\hline Con24 & $\begin{array}{c}11.47 \\
\mathrm{aA} 1\end{array}$ & 0.21 & $\begin{array}{c}10.75 \\
\mathrm{aB} 1\end{array}$ & 0.06 & $\begin{array}{c}10.85 \\
\mathrm{aB} 1\end{array}$ & 0.16 & $\begin{array}{l}8.55 \\
\text { bB1 }\end{array}$ & 0.79 & $\begin{array}{c}11.03 \\
\mathrm{aB} 1\end{array}$ & 0.15 & $\begin{array}{l}8.32 \\
\text { bB2 }\end{array}$ & 0.95 & $\begin{array}{l}9.15 \\
\text { bB1 }\end{array}$ & 0.14 & $\begin{array}{l}3.28 \\
\mathrm{CC} 2\end{array}$ & 0.27 \\
\hline Hay2 & $\begin{array}{l}0.00 \\
\mathrm{dC} 1\end{array}$ & 0.00 & $\begin{array}{l}14.07 \\
\text { bcA1 }\end{array}$ & 0.09 & $\begin{array}{l}14.67 \\
\text { abA1 }\end{array}$ & 0.35 & $\begin{array}{c}15.93 \\
\text { aA2 }\end{array}$ & 0.38 & $\begin{array}{c}15.50 \\
\mathrm{aA} 2\end{array}$ & 0.40 & $\begin{array}{c}15.83 \\
\mathrm{aA} 1\end{array}$ & 0.19 & $\begin{array}{c}15.73 \\
\mathrm{aA} 1\end{array}$ & 0.28 & $\begin{array}{c}13.17 \\
\text { CA1 }\end{array}$ & 0.52 \\
\hline Hay8 & $\begin{array}{c}16.39 \\
\mathrm{aA} 1\end{array}$ & 1.27 & $\begin{array}{l}9.00 \\
\text { cdB1 }\end{array}$ & 0.62 & $\begin{array}{c}11.80 \\
\text { bB2 }\end{array}$ & 0.34 & $\begin{array}{l}9.27 \\
\mathrm{cB} 1\end{array}$ & 0.30 & $\begin{array}{l}9.47 \\
\mathrm{CC} 1\end{array}$ & 0.36 & $\begin{array}{l}8.88 \\
\text { cdB1 }\end{array}$ & 0.36 & $\begin{array}{l}7.80 \\
\mathrm{~dB} 1\end{array}$ & 0.21 & $\begin{array}{l}6.05 \\
\text { eB1 }\end{array}$ & 0.20 \\
\hline Hay24 & $\begin{array}{c}11.52 \\
a B 1\end{array}$ & 0.18 & $\begin{array}{c}10.07 \\
\text { bB1 }\end{array}$ & 0.13 & $\begin{array}{l}10.28 \\
a b C 1\end{array}$ & 0.31 & $\begin{array}{l}8.62 \\
\text { cB1 }\end{array}$ & 0.65 & $\begin{array}{l}10.82 \\
\text { abB1 }\end{array}$ & 0.14 & $\begin{array}{l}8.65 \\
\text { CB12 }\end{array}$ & 0.71 & $\begin{array}{l}7.63 \\
\mathrm{CB} 2\end{array}$ & 0.13 & $\begin{array}{l}1.98 \\
\mathrm{dC} 1\end{array}$ & 0.53 \\
\hline
\end{tabular}

$\mathrm{LSD}=1.26$

*: Feed averages (within each row; for the same feed and same time period) with different small letters denote statistically significant differences ( $p<0.05$ )

: Time period averages (within each column; for the same feed group and same supplement group) with different capital letters denote statistically significant differences $(p<0.05)$

: Feed averages (within each column; for the same supplement group and same time periods) with different numbers denote statistically significant differences ( $p<0.05$ ) 


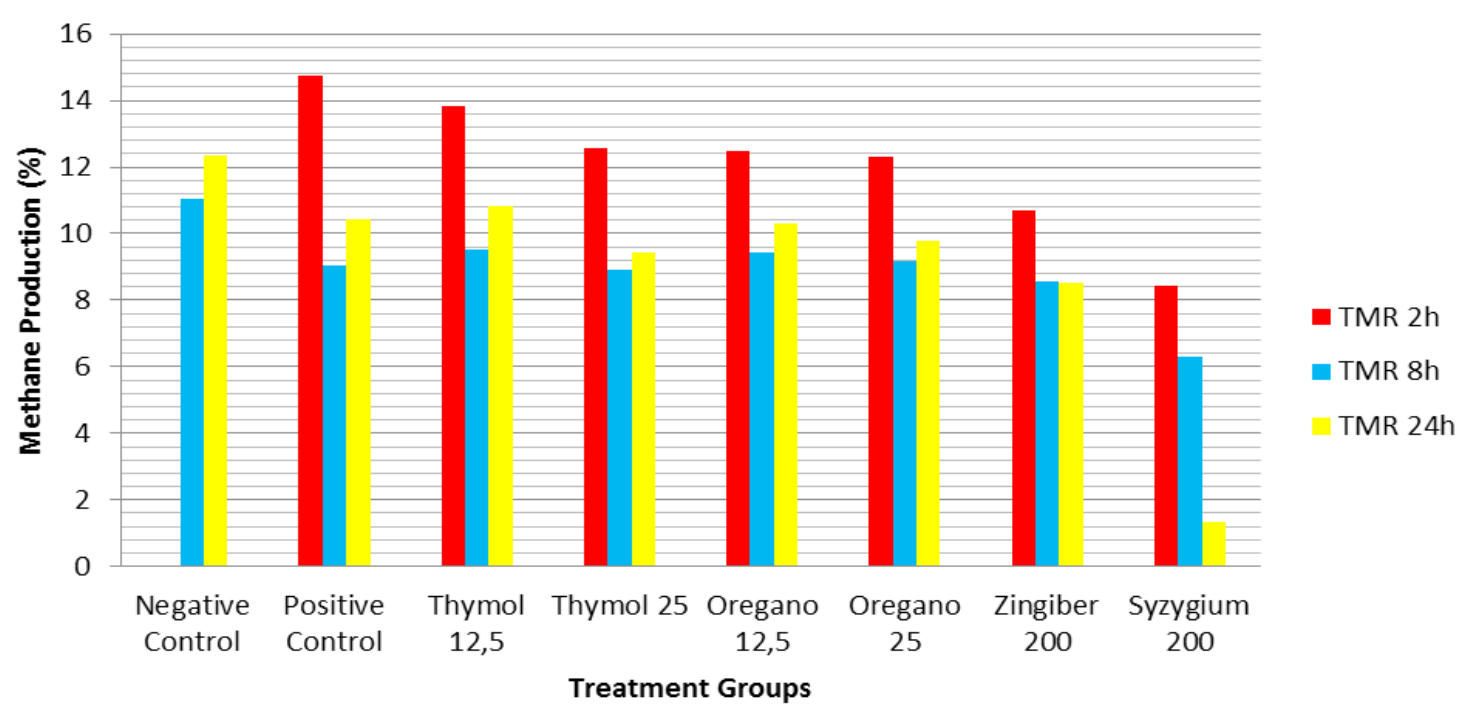

Figure 1. Methane Production (\%) for TMR Treatment Groups

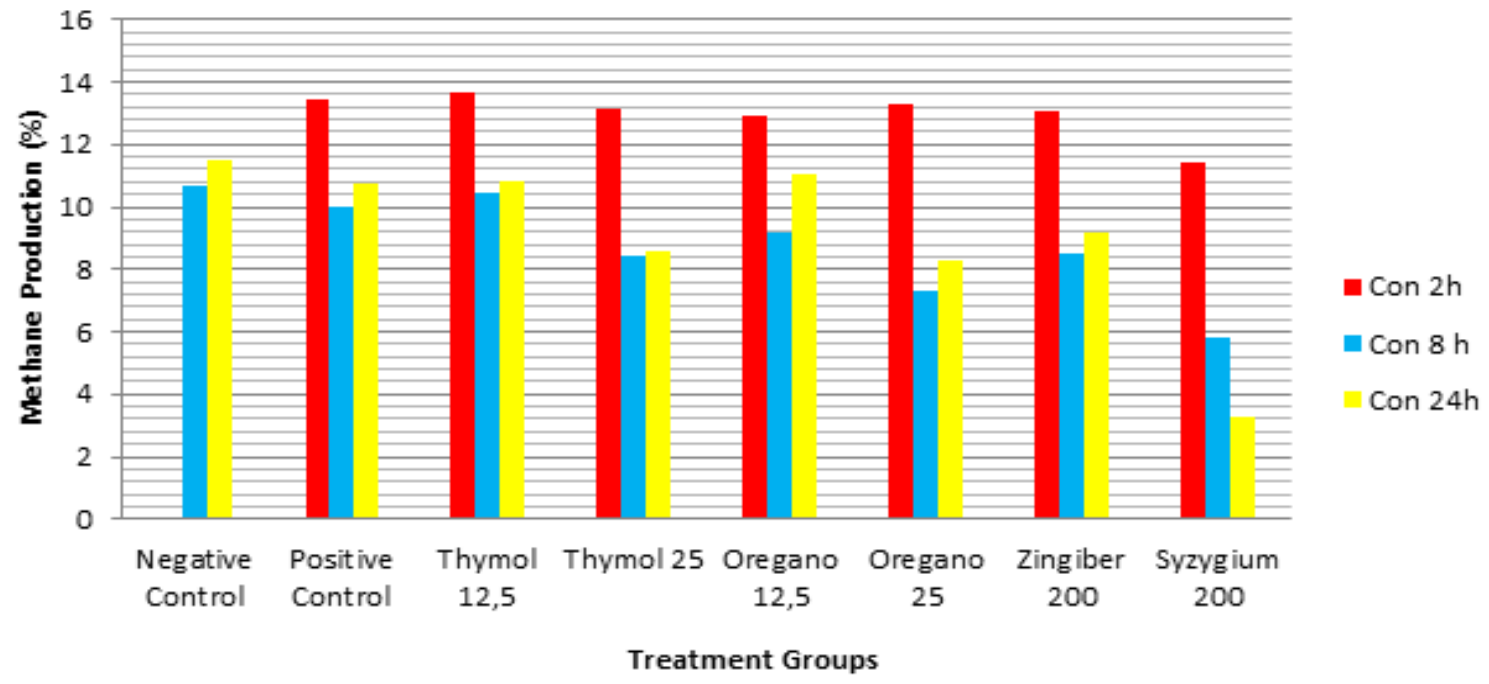

Figure 2. Methane production (\%) for concentrate treatment groups

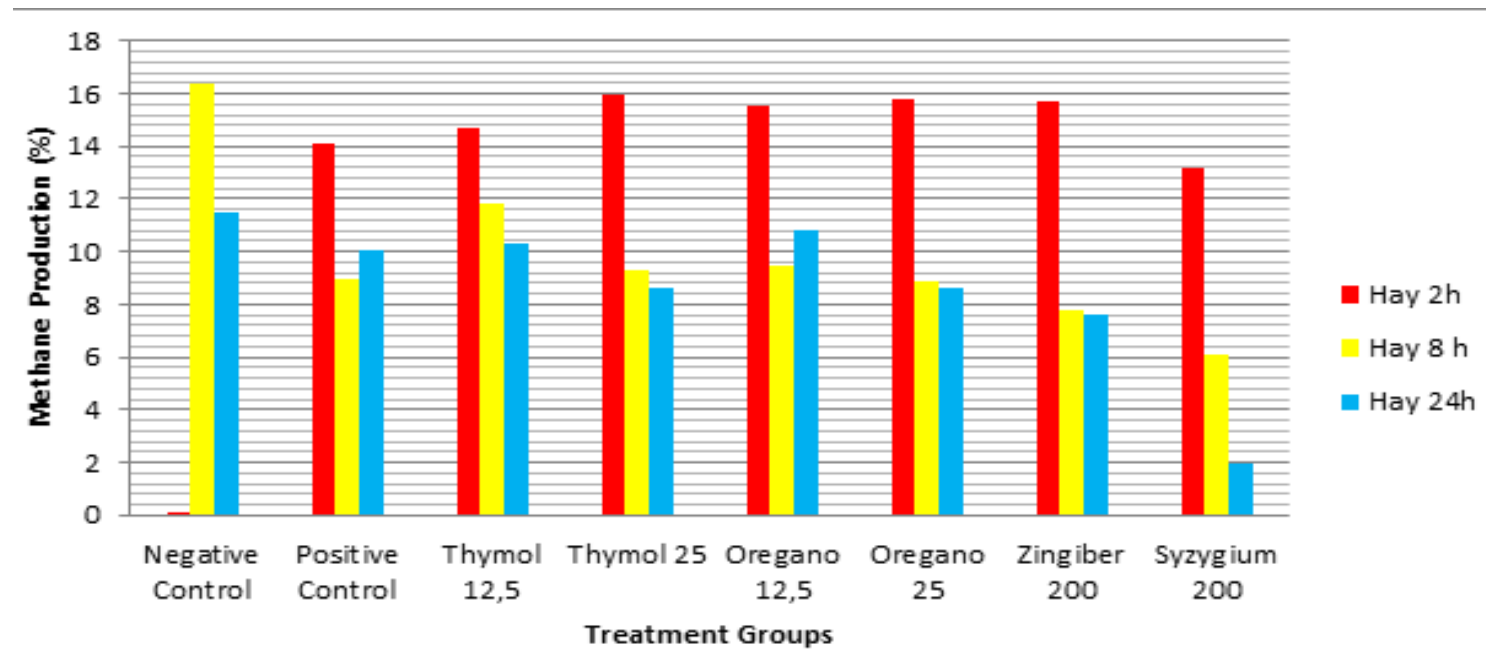

Figure 3. Methane production (\%) for hay treatment groups 
groups at $24 \mathrm{~h}$ were respectively 12.34 and 10.45 while the lowest methane production value (1.65) was recorded in the TMR group with Syzygium 200 supplementation $(p<0.05)$. For concentrate group, methane production values at $24 \mathrm{~h}$ significantly decreased with Thymol 25, Oregano 25, Zingiber 200 and Syzygium 200 supplementations compared to negative control and positive control groups $(p<0.05)$.

The lowest methane production value (3.28) for concentrate group was recorded with Syzygium 200 supplementation at $24 \mathrm{~h}$ $(p<0.05)$ as similarly observed for the TMR. For hay group, methane production values at $24 \mathrm{~h}$ significantly decreased with Thymol 25, Oregano 25, Zingiber 200 and Syzygium 200 supplementations compared to negative control and positive control groups. The lowest methane production value (1.98) for hay group was recorded with Syzygium 200 supplementation at $24 \mathrm{~h}$ as observed for TMR and concentrate substrates. Syzygium 200 supplementation resulted $84.21 \%, 69.49 \%$ and $80.34 \%$ reduction in methane production at $24 \mathrm{~h}$ in TMR, concentrate and hay groups compared to the positive control group, respectively. As indicated by the findings of the study, essence oils have significant effect on reduction of methane production and this effect becomes more apparent at higher doses of essence oil supplementations.

\section{DISCUSSION}

Effects of essence oils on methanogenesis. Methanogenic (methane producing) bacteria convert $\mathrm{H}_{2}$ and $\mathrm{CO}_{2}$ into methane. In the rumen, formation of methane is the major way of hydrogen elimination through the following reaction (12):

$\mathrm{CO}_{2}+4 \mathrm{H}_{2} \rightarrow \mathrm{CH}_{4}+2 \mathrm{H}_{2} \mathrm{O}$

Methanogens reduce hydrogen and carbondioxide to produce methane and meet their energy requirement. The main methanogens in rumen microbial ecosystem are Methanobrevibacter ruminantium, Methanomicrobium mobile and Methanosarcina. Using high starch concentrate feed instead of roughage reduces methane production, energy loss and global pollution (13). The loss of feed energy as methane in mature ruminants depending on ration composition ranges between $2-12 \%$ gross
El menor valor de producción de metano (3.28) por grupo de concentrado se observó en la suplemetación con Syzygium 200 a las 24 h $(p<0.05)$, que se observó de manera similar en TMR. En cuanto al grupo de heno, los valores de producción de metano a las $24 \mathrm{~h}$ disminuyeron significativamente con la suplementación de Thymol 25 , Oregano 25, Zingiber 200 y Syzygium 200 en comparación con los grupos de control negativo y control positivo. El menor valor de producción de metano (1.98) por grupo de heno se observó tanto en la suplemetación con Syzygium 200 a las $24 \mathrm{~h}(\mathrm{p}<0.05)$ como en sustratos de concentrado en TRM. La suplementación con Syzygium 200 dio como resultado reducciones del $84.21 \%, 69.49 \%$ y $80.34 \%$ en la producción de metano a las 24 $h$ en los grupos de TMR, concentrado y heno, respectivamente, al ser comparados con el grupo de control positivo. Como lo indican los resultados del estudio, los aceites esenciales tienen un efecto significativo sobre la reducción de producción de metano, efecto que se hace se hace más evidente en dosis más altas de suplementación con aceites de esencias.

\section{DISCUSIÓN}

Efectos de los aceites de esencias sobre la metanogénesis. Las bacterias metanogénicas (productoras de metano) convierten el $\mathrm{H}_{2}$ y $\mathrm{CO}_{2}$ en metano. En el rumen, la formación de metano es la principal forma de eliminar hidrógeno por medio de la siguiente reacción (12):

$\mathrm{CO}_{2}+4 \mathrm{H}_{2} \rightarrow \mathrm{CH}_{4}+2 \mathrm{H}_{2} \mathrm{O}$

Los metanógenos reducen el hidrógeno y el dióxido de carbono para producir metano y satisfacer sus necesidades energéticas. Los principales metanógenos en el ecosistema microbiano del rumen son Methanobrevibacter ruminantium, Methanomicrobium mobile y Metha-nosarcina. El uso de concentrado con altos contenidos de almidón en lugar de forrajes reduce la producción de metano, la pérdida de energía y la contaminación global (13). La pérdida de energía alimentica en rumiantes maduros en la forma de metano, según la composición de la ración, oscila entre el $2-12 \%$ de la ingesta bruta de energía alimentaria (14). El hidrógeno en el rumen se produce como resultado de la fermentación de nutrientes bajo condiciones anaeróbicas. Este hidrógeno se utiliza en la síntesis de ácidos grasos volátiles y proteínas microbianas (15).

Se liberan mayores cantidades de hidrógeno durante la producción microbiana de ácido acético y butírico. El hidrógeno es utilizado por las bacterias metanogénicas y se convierte en metano con 
feed energy intake (14). Hydrogen in rumen is produced as a result of fermentation of nutrients in anaerobic conditions. This hydrogen is used in the synthesis of volatile fatty acids and microbial proteins (15).

Higher amount of hydrogen is released during microbial production of acetic and butyric acid. Hydrogen is utilized by methanogenic bacteria and converted into methane with $\mathrm{CO}_{2}$ (16). In contrary to the process in acetic and butyric acid, ambient hydrogen ions are utilized during the production of propionic acid. Acetic and butyric acid can not be used in gluconeogenesis like propionic acid. Hence, conversion of volatile fatty acid profile in favour of propionate will ensure more efficient use of feed energy (16).

Protozoa produce much hydrogen as with gram positive bactaria in rumen and increases methane production by creating a symboitic relationship with methanogenic bacteria. Although protozoa have useful effects, decreasing of them in number affects productivity in ruminants in a positive way (16). It has been reported that extract of Myristica fragrans significantly reduces the number of protozoa. Decreasing of protozoa number, increasing of bacteria, fungi number, increasing of propionate production and decreasing of methanogenes improve performance in ruminants (15). Energy loss in rumen fermentation (methane) can limit production performance and can also lead to environmental pollution (1).

As seen in table 2 , methane production rates significantly decreased in time in all treatment groups for all feeds $(p<0.05)$. Methane production rates, depending on time period, type of essence oil and dose amount, ranged between 13.85 and 1.65 for TMR, between 13.67 and 3.28 for concentrate, and between 15.93 and 1.98 for hay. The lowest methane production rates at $24 \mathrm{~h}$ for TMR, concentrate and hay are recorded respectively as 1.65 , 3.28 and 1.98 in the group with Syzygium 200 supplementation $(p<0.05)$.

Syzygium 200 supplementation resulted $84.21 \%, 69.49 \%$ and $80.34 \%$ reduction in methane production at $24 \mathrm{~h}$ in TMR, concentrate and hay groups, respectively. Pen et al (17) reported that while Yucca schidigera extract, depending on dose, resulted upto $42 \%$ reduction in methane production, Quillaja saponaria extract had no effect on methane production. Bodas et al (18) demonstrated that Cardus pynocephalus, Populus tremula, el $\mathrm{CO}_{2}$ (16). A diferencia de los procesos con ácido acético y acido butírico, se utilizan iones de hidrógeno ambiente durante la producción de ácido propiónico. Los ácidos acético y butírico no pueden ser utilizados en la gluconeogénesis, a diferencia del ácido propiónico. Por lo tanto, la conversión del perfil de ácidos grasos volátiles en favor del propionato garantizará un uso más eficiente de la energía alimentaria (16).

Los protozoos producen mucho hidrógeno como lo hace la bactaria gram positiva en el rumen y aumentan la producción de metano creando una relación simbiótica con las bacterias metanogénicas. Aunque los protozoos tienen fines útiles, al disminuir su cantidad se afecta positivamente la productividad de los rumiantes (16). Ha sido reportado que el extracto de Myristica fragrans reduce significativamente el número de protozoos. La disminución en el número de protozoos, el aumento en la cantidad de bacterias, la cantidad de hongos, el aumento en la producción de propionato y la disminución de los metanógenos mejoran el rendimiento de los rumiantes (15). La pérdida de energía en la fermentación ruminal (metano) puede limitar el rendimiento de la producción y puede contribuir a la contaminación ambiental (1).

Como lo muestra la Tabla 2, los valores de producción de metano disminuyeron significativamente en el tiempo a lo largo de todos los grupos de tratamiento y con todos los piensos $(p<0.05)$. Las tasas de producción de metano, de acuerdo al período de tiempo, el tipo de aceite de esencia y la cantidad de dosis, oscilaron entre 13.85 y 1.65 en TMR, entre 13.67 y 3.28 en concentrado y entre 15.93 y 1.98 en heno. Las menores tasas de producción de metano a las $24 \mathrm{~h}$ para TMR, concentrado y heno se registran respectivamente en $1.65,3.28$ y 1.98 en el grupo suplementado con Syzygium $200(p<0.05)$.

La suplementación con Syzygium 200 dio como resultado reducciones del $84.21 \%, 69.49 \%$ y $80.34 \%$ en la producción de metano a las 24 $h$ en los grupos de TMR, concentrado y heno, respectivamente. Pen et al (17) reportaron que mientras que el extracto de Yucca schidigera, según la dosis, llevó a una reducción en la producción de metano del $42 \%$, el extracto de Quillaja saponaria no surtió ningún efecto sobre la producción de metano. Bodas et al (18) demostraron que Cardus pynocephalus, Populus tremula, Prunus avium, Quercus robur, Rheum nobile y Salix suplementación caprea a 50:40:10 alfalfa: heno: cebada en raciones de ovejas disminuyó significativamente la producción de metano a las $24 \mathrm{~h}$ de incubación in vitro. Bunglavan et al (19) reportaron que los extractos a base de agua de rizomas de Zingiber officinale suplementados con raciones de forraje: concentrado en una proporción de 60:40 redujeron 
Prunus avium, Quercus robur, Rheum nobile and Salix caprea supplementation to 50:40:10 alfalfa:hay:barley sheep rations significantly decreased methane production at $24 \mathrm{~h}$ of incubation in in vitro conditions. Bunglavan et al (19) reported that waterbased extracts of Zingiber officinale rhizomes supplemented to 60:40 roughage:concentrate rations significantly decreased methane production. Ahari et al (10) observed that thyme supplementation ( 0.25 and 0.50 $\mathrm{ml}$ ) to $100 \%$ roughage (alfalfa) rations significantly decreased methane production at $4,12,16,24,48,72$ and 96 h in in vito conditions. Canpolat et al (20) demonstrated that carvacrol supplementation significantly decreased methane production. Goel et al (14) added 1, 2 and $3 \mathrm{ml}$ of Cinnamomum zeylanicum (Cinnamon) extracts to sorghum based rations including low, medium and high doses of cellulose. They observed that $3 \mathrm{ml}$ of extract supplementation to the rations with high doses of cellulose resulted reduction in methane production at maximum level $(45.37 \%)$.

Sallam et al (21) reported that essence oils extracted from Achillea santolina (75 $\mu \mathrm{l})$, Artemisia judaica (75 $\mu \mathrm{l}$ ) and Mentha microphylla $(25,50,75 \mu l)$ significantly suppressed methane production at $24 \mathrm{~h}$. Shabestari et al (7) demonstrated that clove methanol extract (CME) did not have any effect on reduction of methane production during early hours ( 2 and $4 \mathrm{~h}$ ) of incubation, but CME significantly reduced methane production in later stages of incubation both in roughage and barley based rations. Patra and Yu (22) reported that increased doses of clove oil, eucalyptus oil, garlic oil, origanum oil and peppermint oil supplementations reduced methane production respectively by $34.3 \%$, $17.6 \%, 42.3 \%, 87.0 \%$ and $25.7 \%$. Santra et al (23) revealed that among 96 different extracts (water, methanol and ethanol) of 32 aromatic plants, 29 plant extracts significantly reduced methane production by $20 \%$ and over. Sirohi et al (24) showed that Myristica fragrans extract resulted upto $48 \%$ reduction in methane production compared to negative control group and they observed significant reduction in number of methanogens $(p \leq 0.05)$. Tekippe et al (25) examined use of essence oils as natural products for suppression of methane production in cattle under in vitro conditions. According to their findings, lavender (Lavandula latifolia) essence oil reduced methane production by $20-30 \%$ and Oregano (Oreganum vulgare) essence oil reduced methane production by $31 \%$. significativamente la producción de metano. Ahari et al (10) observaron que la suplementación con tomillo $(0,25$ y $0,50 \mathrm{ml})$ en raciones de $100 \%$ de forraje, las raciones de (alfalfa) redujeron significativamente la producción de metano a 4, 12, 16, 24, 48, 72 y $96 \mathrm{~h}$ en condiciones in vitro . Canpolat et al (20) demostraron que la suplementación con carvacrol redujo significativamente la producción de metano. Goel et al (14) añadieron 1, 2 y $3 \mathrm{ml}$ de extractos de Cinnamomum zeylanicum (canela) a raciones de a base de sorgo, incluyendo dosis de celulosa bajas, medias y altas. Observaron que con $3 \mathrm{ml}$ de suplementos de extractos en raciones con altas dosis de celulosa dieron como resultado una reducción en la producción de metano al máximo nivel (45.37\%).

Sallam et al (21) informaron que los aceites de esencia extraídos de Achillea santolina (75 $\mu \mathrm{l})$, Artemisia judaica $(75 \mu \mathrm{l})$ y Mentha microphylla $(25,50,75 \mu \mathrm{l})$ suprimieron significativamente la producción de metano a las $24 \mathrm{~h}$. Shabestari et al (7) demostraron que el extracto de clavo de metanol (CME) no tuvo ningún efecto en reducir la producción de metano durante las primeras horas ( 2 y $4 \mathrm{~h}$ ) de incubación, pero el CME redujo significativamente la producción de metano en etapas posteriores de incubación, en raciones a base de forraje y de cebada. Patra y Yu (22) reportaron que un aumento en las dosis de aceite de clavo, aceite de eucalipto, aceite de ajo, aceite de origanum y aceite de menta en la suplementación redujo la producción de metano, respectivamente, en un $34.3 \%$, $17.6 \%, 42.3 \%, 87.0 \%$ y $25.7 \%$. Santra et al (23) revelaron que entre 96 extractos (agua, metanol y etanol) distintos de 32 plantas aromáticas, 29 extractos de plantas redujeron significativamente la producción de metano en un $20 \%$ y más. Sirohi et al (24) demostraron que el extracto de Myristica fragrans produjo una reducción en la producción de metano de hasta un $48 \%$ en comparación con el grupo control negativo y se observó una reducción significativa en la cantidad de metanógenos ( $p \leq 0.05)$. Tekippe et al (25) estudiaron el uso de aceites de esencias como productos naturales para suprimir la producción de metano en bovinos bajo condiciones in vitro. Según sus hallazgos, el aceite de esencia de lavanda (Lavándula latifolia) redujo la producción de metano en un $20-30 \%$ y el aceite esencial de orégano (Oreganum vulgare) redujo la producción de metano en un $31 \%$.

Bhatta et al (8) informaron que las plantas aromáticas con contenido de taninos tales como Clerodendrum inerme, Gymnema sylvestre y Sapindus laurifolia tienen el potencial de suprimir la metanogénesis in vitro. Chaudhary y Gupta (26) demostraron que la semilla de Melia azadiracta y los aceites de esencias de $S$. aromaticum disminuyeron la producción de metano respectivamente en un $27.7 \%$ y un $29.34 \%$ en comparación con el control. 
Bhatta et al (8) reported that aromatic plants with tannin content such as Clerodendrum inerme, Gymnema sylvestre and Sapindus laurifolia have the potential to suppress in vitro methanogenesis. Chaudhary and Gupta (26) demonstrated that Melia azadiracta seed and $S$. aromaticum essence oils reduced methane production respectively by $27.7 \%$ and $29.34 \%$ compared to control. Schizium cumin essence oil was found to reduce methane production by $5.09 \%$. The essence oils Delbergia sisso leave, Schizium cumin bark, Emblica officinalis fruit, Melia azadiracta seed, Allium cepa bulb, Psidium guajava, Azadiracta indica leave, Syzygium aromaticum were reported to reduce methane production by over $15 \%$. Oskoueian et al (27) showed that flavonoids significantly reduce methane production in cattle without inducing any negative effect on rumen microbial fermentation. Kim et al (28) determined in in vitro study carried out in ruminants that medical plant exract of Arisaema ringens decreases methane production more than 43 percent for 24 hours. The findings of these researchers support the findings of the present study. As indicated by research findings, essence oils have significant effect on reduction of methane production and this effect becomes more apparent at higher doses of essence oil supplementations.

In contrary to the current study, Storlien et al (29) reported that supplementation of hop and steroidal saponin extracts in barley-based $(47 \%)$ and barley silage-based $(43 \%)$ rations did not reduce methane production under in vitroconditions. Moreover, combined use of these extracts was found to significantly increase methane production. Al-Hadeethi et al (30) reported that 20 and $30 \mathrm{~g} / \mathrm{kg}$ DM doses levels of exrtracts of green tea (Camellia sinensis), turmeric (Curcuma longa) and garlic do not have a remerkable effect on formation of rumen methane. This case can be associated with the fact that avaliable additives do not create an important effect on rumen fermentation. Such differences in research findings can be attributed to the differences in ration composition, plant extracts used, extraction methods and dose amounts.

It can be concluded that essence oil supplementations to ruminant rations have noteworthy potential of reducing greenhouse gases that cause global warming. Reduced methane production resulted by essence oil supplementations can be attributed to:1) possible inhibition of methanogenic bacteria due to strong antimicrobial effects of essence oils against gram positive and
Se encontró que el aceite de esencia de Comino de Schizium reduce la producción de metano en un $5.09 \%$. Se reportó que los aceites esenciales de hoja de Delbergia sisso, la corteza de Comino Schizium, el fruto de Emblica officinalis, la semilla de Melia azadiracta, el bulbo de Allium cepa, Psidium guajava, la hoja de Azadiracta indica, Syzygium aromaticum redujeron la producción de metano en más del $15 \%$. Oskoueian et al (27) demostraron que los flavonoides reducen significativamente la producción de metano en el ganado sin inducir ningún efecto negativo en la fermentación microbiana ruminal. Kim et al (28) determinaron en un estudio in vitro realizado en rumiantes que el extracto de la planta medicinal Arisaema ringens disminuye la producción de metano en más de un 43 por ciento durante 24 horas. Los hallazgos de estos investigadores respaldan los hallazgos del presente estudio. Como lo indican los resultados del estudio, los aceites de esencias tienen un efecto significativo sobre la reducción en la producción de metano, efecto que se hace se hace más evidente en dosis más altas de suplementación con aceites de esencia.

Contrario al presente estudio, Storlien et al (29) informaron que la suplementación a base de extractos de lúpulo y saponina estroidal en raciones a base de cebada (47\%) y ensilado de cebada (43\%) no redujo la producción de metano en condiciones in vitro. Además, se encontró que el uso combinado de estos extractos aumentó significativamente la producción de metano. AlHadeethi et al. (30) reportaron que dosis de $20 \mathrm{y}$ $30 \mathrm{~g} / \mathrm{kg}$ de DM de extractos de té verde (Camellia sinensis), cúrcuma (Cúrcuma longa) y ajo no tienen un efecto significativo en la formación de metano ruminal. Este caso puede estar asociado al hecho de que que los aditivos disponibles no tienen un efecto de importancia en la fermentación ruminal. Estas diferencias entre los hallazgos de investigación se pueden atribuir a las diferencias en la composición de raciones, en los extractos vegetales utilizados, en los métodos de extracción y en la dosificación.

Es posible concluir que la suplementación con aceites de esencias en las raciones de rumiantes tiene un potencial notable en la reducción de los gases de efecto invernadero, causantes del calentamiento global. La reducción en la producción de metano como resultado del uso de suplementos de aceite de esencias puede atribuirse a: 1) una posible inhibición de las bacterias metanogénicas debido a los fuertes efectos antimicrobianos de los aceites esenciales contra las bacterias gram positivas y gram negativas; y 2) una reducción en la producción de metano por el cambio de protones $\mathrm{H}_{2}$ a la producción de priopinato, lo que impide la reacción de $\mathrm{CO}_{2}$ con $\mathrm{H}_{2}$, dando como resultado una reducción en la producción de metano. La 
gram negative bacteria; and 2 ) reduction of methane production by the shift of $\mathrm{H}_{2}$ protons to propionate production which will prevent reaction of $\mathrm{CO}_{2}$ with $\mathrm{H}_{2}$ and result in reduced methane production. Energy loss as methane not only worsens animal performance, but also increases environmental pollution.

One of the most important goals of stock breeders is manipulation of rumen microbial ecosystem to improve ruminant performance and to reduce methane emissions generated by ruminants. There are several methods to reduce methane emission in rumen. One of these methods is the use of essence oils extracted from aromatic plants. In the prsent study, among all plant extracts, Syzygium 200 ppm supplementation resulted the lowest methane production values. Also, Thymol 25, Oregano 25, Zingiber 200 and Syzygium 200 ppm supplementations significantly decreased methane production for TMR, concentrate and hay with increased durations.

In conclusion it has been concluded that increasing doses of essence oil highly decreases methane production in ruminants. Essence oils can be used as alternatives to chemical supplements (such as anti-biotic, ionophore) used for rumen regulators. Being natural, these substances are environmental friendly. Reduction of methane production is internationally considered as important for mitigating global warming, hence, this study produces highly valuable outputs in this regard.

\section{Acknowledgements}

The authors are grateful to Hohenheim University (Germany) for its financial and technical support. pérdida de energía en forma de metano empeora el rendimiento animal, además de aumentar la contaminación ambiental.

Uno de los más importantes objetivos de los ganaderos es la manipulación del ecosistema microbiano ruminal para mejorar el rendimiento en rumiantes y reducir las emisiones de metano generadas por los rumiantes. Existen varios métodos para reducir la emisión de metano en el rumen. Uno de estos métodos es el uso de aceites de esencias extraídos de plantas aromáticas. En el presente estudio, entre todos los extractos vegetales, la suplementación con Syzygium 200 ppm produjo los valores más bajos en la producción de metano. Además, los suplementos de Thymol 25, Orégano 25, Zingiber 200 y Syzygium 200 ppm redujeron significativamente la producción de metano en TMR, concentrado y heno con un aumento de duración paulatino.

En conclusión, se concluye que el aumento en las dosis de aceite de esencias disminuye en gran medida la producción de metano en rumiantes. Los aceites de la esencias se pueden utilizar como alternativa a los suplementos químicos (tales como anti-biotic, ionophore) utilizados para los reguladores ruminales. Al ser éstas sustancias naturales, son amigables con el medio ambiente. La reducción de la producción de metano es considerada como un factor importante para mitigar el calentamiento global a nivel internacional, por lo que el presente estudio produce resultados muy valiosos a ese respecto.

\section{Agradecimientos}

Los autores agradecen a la Universidad de Hohenheim (Alemania) por su apoyo financiero y técnico.

\section{REFERENCES}

1. Calsamiglia S, Busquet M, Cardoza PW, Castillejos L, \& Ferret A. Invited Review: Essential oils as modifiers of rumen microbial fermentation. J Dairy Sci 2007; $90(6): 2580-2595$.

2. Goel N, Sirohi SK, Dwivedi J. Studies on the effects of methanolic extract of Cinnamomum zeylanicum on in vitro methane inhibition and rumen fermentation patterns. J Chem Pharm Res 2011; (3):609-615.
3. Hart KJ, Yanez-Ruiz DR, Duval, SM, McEwan NR, Newbold CJ. Plant extracts to manipulate rumen fermmentation. Anim Feed Sci Technol2008; 147(1-3):8-35.

4. Takahashi J. Some prophylactic options to mitigate emission from animal agriculture in Japan. Asian-Aust J Anim Sci 2011; 24(2):285-294. 
5. López S, Makkar HPS, Soliva CR. Screening plants and plant products for methane inhibititors. In: Vercoe PE, Makkar HPS, Schlink AC. (ed.). In vitro screening of plant resources for extra nutritional attributes in ruminats: Nuclear and related methodologies. New York: Springer Publications; 2010.

6. Takashi J. Lactic acid bacteria and mitigation of GHG emission from ruminant livestock. In: Marcelino K. (ed.). Lactic acid bactaria R\&D fro food, health and livestock purposes. In Tech Publications; 2013.

7. Shabestari $A H$, Salamatdoustnobar $R$, MaheriI-Sis N, Gorbani A, Ahari KM, Noshadi A, Samadi H, Nezhad JS. Evaluation effects of Clove methanol extract on Methane production in the In vitro condition. Pakistan J Nutr2011; 10(12):1154-1157.

8. Bhatta $R$, Baruah L, Saravanan M, Suresh KP, Sampath KT. Effect of medicinal and aromatic plants on rumen fermentation, protozoa population and methanogenesis in vitro. J Anim Physiol Anim Nutr2013; 97(3):446-456.

9. Faostat, FAO statisticaldatabase. [en linea] 2014. [acceso febrero de 2017]. URL Disponible en: http://faostat. fao.org/site/573/DesktopDefault. aspx?PageID $=573$ \# ancor

10. Ahari KM, Salamatdoustobar R, Maheri-Sis N, Gobrani A, Shabestari AH, Noshadi A, Samadi $H$, Nezhad JS. Effects of the Thyme Extract on the ruminal methane production. Pakistan J Nutr 2011; 10(12):1146-1148.

11. Shardt YAW. Statistics for chemical and process engineers a modern approach. Switzerland: Springer Publications; 2015.

12. Priambodo TW. Effects of medium-chain fatty acids and ration type on in vitroruminal methane production. [Phd Thesis]. Bonn, Germany: Rheinische Friedrich Wilhelms Universität Bonn, Institut für Tierwissenschaften; 2015.

13. Alataş MS, Umucalılar HD. Bacteria of The Rumen Ecosystem and Their Roles. J Fac Vet Med Ataturk Univ 2011; 6(1):71-83.

14. Goel N, Sirohi SK, Dwivedi J. Studies on the effects of methanolic extract of Cinnamomum zeylanicum on In vitro Methane inhibition and rumen fermentation patterns. J Chem and Pharma Res.2011; 3(6):609-615.
15. Sirohi SK, Pandey N, Goel N, Singh B, Mohini M, Pandey P,Chaudhry PP. Microbial activity and ruminal methanogenesis as affected by plant secondary metabolites in different plant extracts. Int J Environ Sci Eng 2009; 1(1):52-58.

16. Demirtaş A, Öztürk $H$, Pişkin İ, Demirkıran $D$, Salgırlı Y. Fidancı UR, Emre B. Effects of Rosemary and Sage Extracts on Ruminal Fermentation Using the Rumen Simulation Technique (Rusitec). J Fac Vet Med Istanbul Univ 2011; 37(2):127-134.

17. Pen B, Sar C, Mwenya B, Kuwaki M, Morikawa $\mathrm{R}$, Takahashi J. Effects of Yucca schidigera and Quillaja saponaria extracts on In vitro ruminal fermentation and methane emission. Anim Feed Sci Technol 2006; 129:175-186.

18. Bodas R, López S, Fernández M, GarcíaGonzález R, Wallace RJ, González JS. Phytogenic additives to decrease In Vitro ruminal methanogenesis. Ciheam Options Méditerranéennes. 2009; 85(A):279-283.

19. Bunglavan SJ, Valli C, Ramachandran M, Balakrishnan V. Effect of supplementation of herbal extracts on methanogenesis in ruminants. Livestock Research for Rural Development. 2010; 22(11): 216[en línea] 2010. [acceso april de 2012]. URL Disponible en: http://www.Irrd.org/ Irrd22/11/bung22216.htm

20. Canbolat O, Kalkan H, Karaman S. Filya I. The Effect of Essential Oils on the Digestibility, Rumen Fermentation and Microbial Protein Production. J Fac Vet Med Kafkas Univ. 2011; 17(4):557-565.

21. Sallam SM, Abdelgaleil SA, Bueno IC, Nasser ME, Araujo RC, Abdalla AL. Effect of some essential oils on In vitro Methane emission. Arch Anim Nutr. 2011; 65 (3): 203-214.

22. Patra AK, Yu Z. Effects of essential oils on methane production and fermentation by, and abundance and diversity of, rumen microbial populations. Applied and Environmental Microbiology. 2012; 78(12):4271-4280.

23. Santra A, Saikia A, Baruah KK. Scope of rumen manipulation using medicinal plants to mitigate methane production. J Phcog 2012; 3(2):115-120. 
24. Sirohi SK, Chaudhary PP, Goel N. Effect of inclusion of Myristica fragrans on Methane production, rumen fermentation parameters and methanogens population. Vet World 2012; 5(6):335-340.

25. Tekippe JA, Hristiov AN, Heyler KS, Zheljazkov VD, Ferreira JFS, Cantrell CL, Varga GA. Effects of plants ans essential oils on ruminal in vitro batch culture methane production and fermentation. Can J Anim Sci 2012; 92 (3): 395-408.

26. Chaudhary UB, Gupta A. Effect of different plant extracts on methane and total gas production in goats under in vitro condition. $2^{\text {nd }}$ International Conference and Exhibition on Nutritional Science \& Therapy, Philadelphia USA. [en línea] 2013. [acceso diciembre de 2013]. URL Disponible en: http:// www.omicsgroup.com/conferences/ACS/ conference/download-pdf.php?file $=390$ Speaker-Pdf.pdf
27. Oskoueian E, Abdullah N, Oskoueian A. Effects of flavonoids on rumen fermantation activity, methane production and microbial population. BioMed Res Int 2013; 1-8.

28. Kim ET, Hwang HS, Lee SM, Lee SJ, Lee IID, Lee SK, Oh DS, Lim JH, Yoon HB, Jeong HY, Im SK, Lee SS. Effects of medicinal herb extracts on in vitro ruminal methanogenesis, microbe diversity and fermentation system. Asian Australas J Anim Sci 2016; 29(9): 1280-1286.

29. Storlien TM, Harstad OM, Narvaez N, Wang $Y$, McAllister TA. Effect of different oils and plant extracts on in vitro ruminal methane production. Acta Agric Scand Sect A Anim Sci 2012; 62(4):300-304.

30. Al-Hadeethi A, Günal M, Reyes D, AbuGhazaleh A. The effects of plants extracts on fermantation and methane production under in vitro conditions. Global Journal of Bio-Science and Biotechnology 2016; 5(2): 253-260. 